\title{
Monsieur Couchepin n'aime pas perdre
}

\begin{abstract}
Après l'échec de la deuxième révision de la LAMal, l'avenir de l'assurance sociale et le financement du système de santé en Suisse restent à définir. Nous avons demandé à Yves Guisan, vice-président de la FMH et conseiller national, d'apporter un peu de clarté dans une discussion menée souvent de manière incohérente et partisane.
\end{abstract}

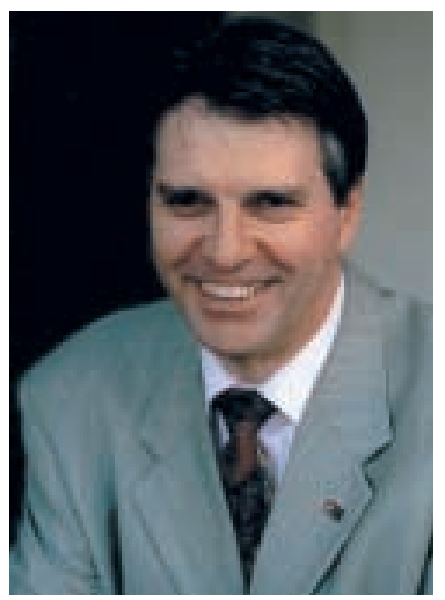

BMS: Le 17 décembre 2003 vous avez, par le biais d'une proposition, fait échouer la deuxième révision de la LAMal. Etes-vous un «empêcheur de danser en rond»?

Dr Yves Guisan: Certainement pas! Mais quand un projet fait manifestement fausse route, quand les idéologies l'emportent sur les réalités, il serait apparu comme totalement incompréhensible voire irresponsable que même les représentants des professions de la santé, en l'occurrence le vice-président de la FMH et les autres médecins élus, y souscrivent alors que des effets pervers dramatiques étaient d'emblée prévisibles. Il ne s'agit là en aucun cas d'un refus au nom d'une conception corporatiste, mais au contraire d'une défense de l'intérêt général en raison d'une perception des réalités du terrain qui échappe à la majorité de Parlement.

La majorité a-t-elle été obtenue par hasard? Cette majorité a effectivement été une surprise. Elle ne s'explique toutefois pas simplement par des absences sur les bancs bourgeois ou le hasard comme certains voudraient le faire entendre. Ces défections étaient d'ailleurs assez uniformément réparties.

La gauche était très fortement déterminée, même jusqu'au referendum, à la suite de l'échec de ses propositions en matière d'objectifs sociaux. Mais cela n'a certainement pas été suffisant en soi. Ce qui a sans doute été déterminant, c'est le manque de conviction face à la perspective certaine de devoir défendre cette mauvaise loi devant le peuple. Ses implications concrètes restrictives à tous les niveaux sans être clairement énoncées (rationnement indirect), tout cela couplé à l'absence de toute garantie quant à une véritable maîtrise des primes, ont manifestement déclenché un malaise suffisamment profond pour faire pencher la balance.

M. Couchepin n'a défendu le projet de révision que du bout des lèvres. Craignait-il le référendum?

Le Conseiller fédéral Couchepin ne partage pas nécessairement nos opinions, mais il a indiscutablement un sens aigu de la politique, même s'il se plante parfois (la retraite à 67 ans!). Le refus de la conférence de conciliation de donner un peu de mou dans le financement des réseaux lui a donné la conviction que cette $2^{\mathrm{e}}$ révision ouvrait trop de fronts simultanés pour passer sans difficulté la rampe d'une votation populaire. Or M. Couchepin n'aime pas perdre. Mais pour gagner il devra accepter que ni la concurrence économique entre fournisseurs de soins, ni les solutions miracles des seuls assureurs, ne stabiliseront la progression des coûts de la santé.

La deuxième révision de la LAMal a été débattue pendant trois ans et rejetée deux fois en votation finale par le Conseil national. Le Conseil des Etats I'a toujours acceptée. Le Parlement serait-il dépassé par la problématique de la santé? Je ne serais pas loin de le penser. Les problèmes de la santé impliquent encore plus que les autres assurances sociales une gestion en fonction de critères humains et non pas exclusivement de chiffres. Le Parlement a énormément de peine à percevoir ces aspects, étant par trop éloigné des problèmes concrets dans le terrain. Les arguments avancés par les professions de la santé sont systématiquement interprétés comme une défense d'intérêts corporatistes. Les clichés ont la vie dure: L'obligation de contracter est comprise comme un revenu assuré des médecins et non pas comme une garantie de remboursement au malade. L'augmentation des coûts est attribuée aux médecins qui multiplient les prestations sans raison et prescrivent inutilement des médicaments coûteux à la place des génériques ... Le Parlement ne perçoit pas ni le poids de la disponibilité nécessaire à la clientèle, ni les difficultés des hôpitaux a trouver du personnel disposé à des horaires irréguliers impliquant un travail de nuit pour des salaires relativement modestes après des formations particulièrement exigeantes. Il ne perçoit pas davantage le parcours du combattant et la dose d'enthousiasme nécessaire pour acquérir une formation de médecin. Les incidences démographiques et les progrès de la médecine sont à 
peine pris en compte. Dans ces conditions des recettes simplistes et parfois contradictoires s'imposent sans réaliser qu'elles aboutissent de facto à un rationnement indirect non dépourvu de conséquences pratiques et éthiques majeures. Le mot rationnement est pourtant banni du vocabulaire tant il est évident que personne n'en veut et que sa seule évocation est une entorse inacceptable à la morale politique ... De toute évidence le Parlement fait davantage confiance à l'opinion publique qu'à ses propres spécialistes.

Il y a donc tout un travail d'information et de sensibilisation à poursuivre inlassablement. Chaque séminaire, chaque intervention dans les médias, la radio et la télévision doit être mise à profit.

Lors de la séance de la Chambre médicale en octobre 2002, on a relevé que le projet de loi était mal conçu, sans concept ni objectif et comportait de nombreuses lacunes juridiques. Le corps médical n'a-t-il rien entrepris et attendu l'élaboration d'un meilleur projet ou n'a-t-il tout simplement pas été entendu?

La FMH a fait tout ce qu'elle pouvait pour être davantage présente. Elle a multiplié les contacts personnels avec les parlementaires et organisé des séances d'information comme le font d'autres groupement d'intérêts. Mais elle n'est pas parvenue ni à motiver des nonmédecins ayant une certaine influence ni à rompre le sentiment profond de méfiance qu'elle inspire. Son discours lors des hearings en commission est apparu comme trop universitaire ou trop syndicaliste. Son discours manque de réalisme politique et se concentre trop exclusivement sur les autorités fédérales et cantonales qui ne sont pas nécessairement les seules bonnes cibles. La FMH doit diriger autant si ce n'est davantage ses efforts en direction des assurés, des consommateurs, des organisations d'aînés, et de handicapés. Cela ne nécessite pas un concept de relations publiques à plusieurs millions, mais du bon sens stratégique et du dévouement. C'est à la FMH qu'il appartient de venir à la rencontre des partenaires sans attendre d'être interpellée. Cette tâche n'est pas la chasse gardée du président et du comité central. Elle doit largement se répartir entre tous les niveaux de responsabilité dans les cantons et dans le terrain. La Société médicale de la Suisse romande (SMSR) en a fourni un premier échantillon avec son manifeste. Il importe absolument que cet effort se généralise et se poursuive.
La FMH a également critiqué le fait que la révision de la loi ne tenait pas compte des besoins de la population. Quels sont ces besoins selon vous? Le projet avait deux défauts rédhibitoires.

Premièrement il érigeait le rationnement indirect en philosophie de base. La clause du besoin, la suppression de l'obligation de contracter, et les réseaux selon la formule du Conseil des Etats respectivement de la conférence de conciliation, cherchaient à limiter l'augmentation du volume de prestations conformément aux propositions simplistes des caisses-maladie. La courbe établissant un parallélisme entre le nombre de cabinets médicaux et l'augmentation des coûts a fait des ravages. Personne n'est prêt à analyser cette question plus en profondeur et admettre que ces conclusions sont manifestement réductrices. Personne ne veut réaliser non plus qu'avec un gel du nombre de cabinets médicaux assorti de contraintes financières et contractuelles ainsi que les multiples contrôles et autres mesures restrictives, une pénurie médicale grave est programmée à brève échéance déjà. Cyniquement dit, rien n'est moins cher que de ne pas soigner les gens en créant des files d'attentes et des engorgements à tous les niveaux. On obtiendrait alors un abaissement des primes au prix de conséquences médicales et sociales désastreuses tout en laissant la responsabilité éthique de cette situation aux professionnels de la santé. Ce sont les conséquences implicites de ces propositions, mais ce discourslà personne ne veut non plus l'entendre. Or il est indispensable de garantir à tous un accès à des soins de qualité.

Deuxièmement les buts sociaux de l'assurance-maladie obligatoire et solidaire ne sont manifestement pas atteints. C'est la cause principale de l'opposition de la majorité qui s'est faite jour le 17 décembre dernier. L'abaissement des primes, en particulier pour les familles avec enfants, présuppose une nouvelle répartition des charges entre pouvoirs publics et contribuables avec à la clé de nouveaux mécanismes de gestion du système. Ce débat de fonds n'a jamais eu lieu et s'est égaré dans des problèmes techniques secondaires ou la disponibilité financière des cantons et de la Confédération.

Dans toute cette opération les besoins de la population avaient manifestement été volontairement oubliés au profit de quelques uns. Une pareille politique ne peut que pénaliser lourdement la croissance de ce pays. Celle-ci ne saurait progresser dans un climat perpétuellement conflictuel et en dehors d'une société relativement homogène qui a fait la force de la Suisse jusqu'à présent. Cette loi se voulait sans le dire 
être un contreprojet indirect à l'initiative de l'UDC qui va encore plus loin. Il est impossible de relancer l'économie et la prospérité en favorisant une société à deux vitesses dont la médecine à deux vitesses n'est que l'une des composantes. La population attend un financement équilibré entre pouvoirs publics et assurés de manière à aboutir à des primes à la portée de la classe moyenne assorti d'un programme social cohérent pour les familles et les plus défavorisés.

\section{Couchepin cherche dorénavant des solutions dans quatre domaines, à savoir: \\ - le financement des hôpitaux; \\ - la compensation des risques; \\ - la limitation du nombre de médecins; \\ - le financement des soins.}

La FMH va-t-elle faire des propositions dans I'un ou l'autre de ces domaines?

Il s'agit de recentrer la révision autour de ces questions fondamentales et de ne pas se laisser piéger par un morcellement des problèmes afin de les éluder en se limitant à des approches techniques. A ce titre un certain nombre de postulats de principes doivent guider la réflexion. Elle doit être placée sous l'égide de l'art. 32 de la LAMal actuelle concernant la qualité, l'efficacité du traitement et son économicité. L'assurance sociale doit être gérée selon les principes du New Public Management. Dans ce cadre le «bench marking» joue un rôle non pas dans la perspective de gains de parts de marché, mais de l'optimisation de l'attribution des ressources. Les indicateurs à prendre en considération ne se limitent pas aux seuls aspects financiers. L'efficacité et la qualité doivent être également au premier plan.

Il s'agit là d'un défi de la plus grande envergure. Certes il ne résoudra pas tous les problèmes. C'est pourtant la seule alternative possible pour garder au centre des préoccupations la dimension humaine et de société. La généralisation de l'Evidence-based Medicine dans la pratique courante postule des changements structurels et surtout de mentalité absolument considérables. Les assureurs devraient se concentrer sur leur mission sociale et intervenir à titre de partenaires au gré de leurs compétences financières et non pas celui de censeur dont l'objectif primordial est d'abord de chercher à ne pas devoir payer. Une restructuration de la pratique médicale autour de ces trois principes, qualité, efficacité, économicité du traitement dans le cadre de cercles loco-régionaux permet de dégager une perspective tout à fait différente à la satisfaction aussi bien des patients que des professionnels concernés. Cela ne signifie en aucun cas de mettre sur pied une nouvelle forme de supermarché de la santé. Des économies considérables peuvent résulter de cette réorientation et rationalisation de la pratique médicale. Les critères retenus quant au choix de la prise en charge feront appel plus que jamais à la responsabilité des uns et des autres, aussi bien les fournisseurs de prestations que les payeurs tout en permettant une meilleure acceptance. Les questions fondamentales devront faire l'objet d'un débat de société pour ne pas tomber dans l'arbitraire.

Les assureurs ont constitué actuellement un nouveau groupe de pression et de profit dont on ne sait plus très bien quels intérêts il représente si ce n'est les siens. Il ne s'agit en tout cas certainement pas des assurés. La concurrence économique entre assureurs a joué à cet égard un effet des plus pervers par la sélection détournée des risques et les conflits d'intérêts entre activité privée et sociale. Une réorganisation est absolument nécessaire pour démocratiser les caissesmaladie et rendre le pouvoir aux assurés comme c'était le cas dans le cadre de l'assurance mutualiste. Les conseils d'administration doivent être élus par les assurés. Cette prise de responsabilité irait de pair avec une conscience accrue des problèmes qui se posent et la recherche de solution avec une volonté de concertation plutôt que de rapports de force. Cette démocratisation est essentielle pour permettre la conduite d'un véritable débat de société autour de la progression des coûts. Si des mesures limitatives doivent inéluctablement être prises à terme, la décision doit reposer sur un large consensus et ne saurait reposer exclusivement sur les épaules des soignants au chevet du malade faute de moyens pour mettre en œuvre la prise en charge souhaitable.

Ces propositions auront de la peine à séduire les politiciens peu enclins à s'embarquer dans de grandes discussions techniques et philosophiques pour résoudre les problème de fonds, mais plutôt à la recherche de mesures immédiates pour abaisser les primes. On préférera augmenter les franchises, éventuellement en fonction du revenu, plutôt que d'aborder le problème fondamental de la répartition du financement entre contribuables et assurés et de mettre en place un dispositif simple de régulation économique et sociale. A l'inverse de ses prédécesseurs, le Conseiller fédéral Pascal Couchepin a déjà pris publiquement conscience que la maîtrise des coûts ne signifie pas blocage, mais maîtrise d'une augmentation progressive qui de toute manière est inéluctable. Encore faut-il se donner les moyens de gérer cette progression. Cela 
demande de disposer des leviers de commande sur les paramètres déterminants du système: gestion du vieillissement démographique, admission des nouvelles technologies obligatoirement à charge des caisses-maladies, gestion de la demande de prestations. Cela demande aussi une réforme institutionnelle qui clarifie les rapports en Confédération et cantons de manière à ce qu'il y ait véritablement un pilote dans l'avion.

La FMH est-elle considérée comme un interlocuteur sérieux?

Pour qu'elle soit prise sérieusement en considération, il est indispensable qu'elle mette fin à ses dissensions intérieures et qu'elle fasse rapidement des propositions structurées. Chacun doit y avoir sa place, médecine générale et spécialisée, pratique hospitalière publique et privée. La FMH gagnera formidablement en crédibilité en réussissant la mise en place de TARMED, même si cela se passe dans la rogne et la grogne (d'ailleurs largement justifiée tant la complication bureaucratique et logistique du système dépassent finalement l'objectif initial de transparence). Il va falloir savoir dépasser ces difficultés tout en leur apportant les correctifs nécessaires pour s'engager avec détermination dans la mise en place d'un système acceptable de gestion de la qualité-efficacité-économicité du traitement. C'est en soi une révolution de la pratique médicale. Il faut être prêt à changer nos habitudes dans l'intérêt de nos patients et de la société, et en fin de compte de notre profession. Mais cet engagement n'exclut pas de montrer les dents chaque fois que le dialogue partenarial, l'égalité dans les rapports de force, ainsi que l'équité sociale et économique seront menacés. Nous ne saurions en rester au succès d'un referendum avorté.

Interview: Markus Trutmann 